\title{
Driving Interconnected Networks to Supercriticality
}

\author{
Filippo Radicchi ${ }^{*}$ \\ Center for Complex Networks and Systems Research, School of Informatics and Computing, \\ Indiana University, Bloomington, Indiana 47408, USA \\ (Received 20 December 2013; published 22 April 2014)
}

\begin{abstract}
Networks in the real world do not exist as isolated entities, but they are often part of more complicated structures composed of many interconnected network layers. Recent studies have shown that such mutual dependence makes real networked systems potentially exposed to atypical structural and dynamical behaviors, and thus there is an urgent necessity to better understand the mechanisms at the basis of these anomalies. Previous research has mainly focused on the emergence of atypical properties in relation to the moments of the intra- and interlayer degree distributions. In this paper, we show that an additional ingredient plays a fundamental role for the possible scenario that an interconnected network can face: the correlation between intra- and interlayer degrees. For sufficiently high amounts of correlation, an interconnected network can be tuned, by varying the moments of the intra- and interlayer degree distributions, in distinct topological and dynamical regimes. When instead the correlation between intraand interlayer degrees is lower than a critical value, the system enters in a supercritical regime where dynamical and topological phases are no longer distinguishable.
\end{abstract}

DOI: 10.1103/PhysRevX.4.021014

The traditional study of networks as isolated entities has recently been overcome by a more realistic approach that accounts for interactions between networks [1]. Networks in the real world are, in fact, often, if not always, mutually connected: Social networks (e.g., Facebook, Twitter) are coupled because they share the same actors [2]; transportation networks are composed of different layers (e.g., buses, airplanes) with common nodes standing for the same geographic locations [3]; the functioning of communication and power-grid systems depends one on the other [1]. As the properties of an isolated network are not trivially deducible from those of the individual vertices that are part of it, at the same strength, decomposing an interconnected network and studying each component in isolation does not allow one to understand the whole system and its dynamics. Indeed, interconnected networks often exhibit properties that strongly differ from those typical of isolated networks: For example, structural [1] and dynamical [4] transitions that are usually continuous in isolated networks [5] may become discontinuous in interconnected networks. So far, theoretical studies have pointed out that phase transitions in random interconnected networks become abrupt only if the strength (or density) of the

\footnotetext{
*filiradi@indiana.edu.
}

Published by the American Physical Society under the terms of the Creative Commons Attribution 3.0 License. Further distribution of this work must maintain attribution to the author(s) and the published article's title, journal citation, and DOI.
Subject Areas: Complex Systems,

Interdisciplinary Physics,

Statistical Physics interconnections is sufficiently large when compared to the first moment of the strength (or degree) distribution of the whole network $[4,6-9]$. In this paper, we show that the situation is not so simple, but, depending on the combination of basic structural features-strength of interconnections, first two moments of the degree distribution of the entire network, and correlation between intra- and interlayer degrees - an interconnected network explores a complicated scenario where its critical properties can drastically mutate.

In the following, we focus our attention on the case of two arbitrarily interconnected network layers and study the spectral properties of its associated normalized Laplacian [10]. The choice of this matrix is not arbitrary. The normalized Laplacian of a network is, in fact, an object of fundamental importance for the understanding of its structural and dynamical properties, sometimes even more than the adjacency matrix. For example, the spectrum of the normalized Laplacian is used in spectral graph clustering to determine the internal organization of a graph [11,12], and many useful measures, such as graph energy [13], graph conductance and resistance [14], and the Randić index [15], are quantifiable in terms of the eigenvalues of the normalized Laplacian. Also, the normalized Laplacian fully describes the behavior of one of the most important dynamical processes studied in science, from biology [16] to computer science [17], from ecology [18] to finance [19] and physics [20]: random-walk dynamics. The normalized Laplacian is representative for both the classical [10] and the quantum [21] versions of random walk in 
networks, and more, in general, for every time-reversible Markov chain [22].

Let us consider a symmetric and weighted interconnected network formed by two interconnected layers of identical size $N$ whose adjacency matrix $G$ is written in the block form

$$
\begin{aligned}
G & =p G_{\text {in }}+(1-p) G_{\text {out }} \\
& =p\left(\begin{array}{cc}
A & \emptyset \\
\varnothing & B
\end{array}\right)+(1-p)\left(\begin{array}{cc}
\emptyset & C \\
C^{T} & \varnothing
\end{array}\right) .
\end{aligned}
$$

$A, B, C$, and $\emptyset$ are $N \times N$ square matrices. $\emptyset$ is a matrix whose elements are equal to zero. $A$ and $B$ are symmetric matrices, while $C$ is not necessarily symmetric. According to Eq. (1), the adjacency matrix $G$ is a convex linear combination of the intra- and interlayer adjacency matrices $G_{\text {in }}$ and $G_{\text {out }}$, and the parameter $p \in[0,1]$ serves to continuously tune the entire network from a bipartite graph $(p=0)$ to a perfectly decoupled networked system $(p=1)$. The normalized graph Laplacian associated with the adjacency matrix of Eq. (1) is defined as

$$
\mathcal{L}=\mathbb{1}-D^{-1 / 2} G D^{-1 / 2} .
$$

In the definition of $\mathcal{L}, \mathbb{1}$ is the identity matrix, and $D^{-1 / 2}$ is a diagonal matrix whose diagonal elements are equal to the inverse of the square root of the node strengths [10]. Since $G$ is symmetric, all the eigenvalues of $\mathcal{L}$ are real numbers in the range $[0,2]$. For simplicity, we sort them in ascending order such that $0=\nu_{1} \leq \nu_{2} \leq \cdots \nu_{2 N} \leq 2$, and we indicate with $\mathbf{w}_{i}$ the eigenvector associated with $\nu_{i}$. The smallest eigenpair $\left(\nu_{1}, \mathbf{w}_{1}\right)$ is generally said to be "trivial" because it depends only on the strength sequence of the graph. We always have $\nu_{1}=0$ and $\mathbf{w}_{1}^{T}=1 / \sqrt{2 N\langle s\rangle}\left(\sqrt{s_{1}}, \ldots, \sqrt{s_{2 N}}\right)$, with $\langle s\rangle$ the first moment of the strength distribution and $s_{i}$ the strength of the node $i$. When translated into the language of random-walk dynamics, $\mathbf{w}_{1}$ tells us that, independently of the initial conditions and the topology of the network, the stationary probability to find the random walker on a given node is linearly proportional to its strength. The other two external eigenvalues $\nu_{2}$ and $\nu_{2 N}$, and their associated eigenvectors $\mathbf{w}_{2}$ and $\mathbf{w}_{2 N}$, are much more meaningful from the structural and dynamical points of view. The value of $\nu_{2}$ is always positive in graphs composed of a single connected component. The associated eigenvector $\mathbf{w}_{2}$ is typically used in graph clustering to determine the bisection corresponding to the minimum of the normalized cut of the graph [11]. In terms of randomwalk dynamics, such an eigenvector is also very meaningful because the signs of its components define the so-called almost invariant sets of the graph [12,23]. In essence, a random walker spends most its time moving among nodes whose correspondent components in $\mathbf{w}_{2}$ have the same sign, and less frequently jumps between nodes whose correspondent components in $\mathbf{w}_{2}$ have different signs. The largest eigenvalue $\nu_{2 N}=2$ only if the graph is bipartite, while $\nu_{2 N}<2$ in all other cases, and the components of $\mathbf{w}_{2 N}$ identify the bipartite components of the graph. A random walker typically jumps between pairs of nodes that correspond to components of $\mathbf{w}_{2 N}$ with different signs.

To understand the spectral properties of $\mathcal{L}$, we restrict our attention to the case of random network models generated according to the following procedure. Intra- and interlayer degrees of both network layers $A$ and $B$ are, respectively, given by the same identical intra- and interlayer degree sequences $k_{\text {in }}=\left\{k_{1}^{\text {in }}, k_{2}^{\text {in }}, \ldots, k_{N}^{\text {in }}\right\}$ and $k_{\text {out }}=\left\{k_{1}^{\text {out }}, k_{2}^{\text {out }}, \ldots, k_{N}^{\text {out }}\right\}$. The numbers appearing in the two degree sequences are also identical except for their order of appearance. The intra- and interlayer degree distributions are thus identical $P\left(k^{\text {in }}\right)=P\left(k^{\text {out }}\right)=P(k)$, while the alignment between the two sequences regulates the joint probability distribution $P\left(k^{\text {in }}, k^{\text {out }}\right)$ of both network layers. In the construction of the network, internal and external connections are randomly placed with the only constraints of preserving the a priori provided degree sequences and generating network layers composed of a single connected component, so that $\nu_{2}>0$ and $\nu_{2 N}<2$ for every $p \in(0,1)$. It is worthwhile to briefly discuss the meaning, limitations, and justifications of the previous assumptions. The fact that intra- and interlayer connections are randomly placed allows us to say that no substructure is present in layers $A$ and $B$. Although this assumption is not good for real networks that instead often exhibit a modular structure at the single-layer level [24], it is a necessary assumption to concentrate our attention only on the effect of the layer structure on the spectrum of $\mathcal{L}$. The facts that the intra- and interlayer degree distributions are identical and that both network layers have the same degree distribution and joint probability function $P\left(k^{\text {in }}, k^{\text {out }}\right)$ are also nonrealistic assumptions. In real-world interconnected networks, in fact, one should expect the various distributions to be different. Although our general solution requires a much weaker assumption (see the Supplemental Material [25]), here we have decided to impose these constraints for illustrative purposes. The scenario is, in fact, already so rich that having additional symmetries in the system facilitates the explanation of the various behaviors that the system can exhibit.

If we think in terms of random-walk dynamics, our network models are symmetric in two ways. First, the stationary probability to find the random walker in one of the two layers is equal to $1 / 2$. Second, they introduce a symmetry around the point $p=1 / 2$ for the various regimes in which the system can be tuned by varying $p$. Intuitively, we expect the following: (i) For $p<1 / 2$, the network should be in a "bipartite" regime ( $\mathcal{B}$ phase) with the random walker moving more often from one layer to the other, and less frequently diffusing in the same layer. (ii) For $p>1 / 2$, the network should be in a "decoupled" regime ( $\mathcal{D}$ phase) with the random walker more likely diffusing between 
nodes of the same layer, and less likely jumping between layers. (iii) For $p \simeq 1 / 2$, the network should be in an "indistinguishable" regime ( $\mathcal{I}$ phase), with the random walker moving between and among layers with equal probability. Please note that, in the $\mathcal{I}$ phase, the system is topologically and dynamically identical to a single-layer network and thus in a potentially safe state. On the contrary, when layers are structurally and dynamically distinguishable (i.e., $\mathcal{B}$ and $\mathcal{D}$ phases), then the network behaves as an interconnected network and, as such, is exposed to potentially catastrophic failures or anomalous dynamical behavior. Intuitively, one should expect that the network is driven in a continuous way among the different phases as $p$ is varied. In reality, the scenario is much more complicated and appealing.

Let us first focus on the exactly solvable case in which the intra- and interlayer degree sequences of both layers are ordered in the same way $\left(k_{i}^{\text {in }}=k_{i}^{\text {out }}=k_{i}\right.$, for all nodes $i$ ). Please note that in this case, the node strengths equal the node degrees, and thus they do not change with $p$ $\left[s_{i}=p k_{i}^{\text {in }}+(1-p) k_{i}^{\text {out }}=k_{i}\right.$, for all nodes $\left.i\right]$. In addition to the trivial eigenpair, $\mathcal{L}$ always has another eigenpair $\left(\nu^{*}, \mathbf{w}^{*}\right)$ given by

$$
\nu^{*}=2(1-p) \quad \text { and } \quad w_{i}^{*}=c_{i} \sqrt{k_{i}}, \quad \forall i=1, \ldots, 2 N,
$$

where $\quad c_{i}=c \quad$ for $\quad i=1, \ldots, N, \quad c_{i}=-c \quad$ for $i=N+1, \ldots, 2 N$, and $c=1 / \sqrt{2 N\langle k\rangle}$ with $\langle k\rangle$ the first moment of the degree distribution (see the Supplemental Material [25]). Equation (3) tells us that the eigenvector $\mathbf{w}^{*}$ is able to perfectly reflect the layer structure of the system. Such an eigenvector is associated with an eigenvalue that spans the entire range $[0,2]$ and therefore must intersect all the other eigenvalues of $\mathcal{L}$. At each point of intersection, two orthogonal eigenvectors correspond to the same eigenvalue, and thus, in physical terms, each point of intersection corresponds to a degenerate energy level. Such a phenomenology is typical of discontinuous phase transitions [26]. Please note that the intersection between eigenvalues happens in networks of any size and for any degree distribution. The changes between the various phases that we described above are of the same nature: As $p$ is increased, the system moves discontinuously from the $\mathcal{B}$ phase to the $\mathcal{I}$ phase, and from the $\mathcal{I}$ phase to the $\mathcal{D}$ phase. The $\mathcal{B}$ phase can be identified as the regime in which $\nu^{*}$ corresponds to the largest eigenvalue of $\mathcal{L}$. Similarly, the $\mathcal{D}$ phase can be determined as the regime in which $\nu^{*}$ represents the second-smallest eigenvalue of $\mathcal{L}$. Finally, in the $\mathcal{I}$ phase, $\nu^{*}$ is inside the spectrum of $\mathcal{L}$. The values of $p$ for which phase transitions occur can be approximately estimated with the following argument. Numerical computations of the spectrum of $\mathcal{L}$ show that, whereas the eigenvalue $\nu^{*}$ is rapidly changing as a function of $p$, all other eigenvalues vary much more slowly (see the upper panels of Fig. 1). The graph obtained for $p=1 / 2$ can thus be used as a representative for this almost constant behavior. We can then approximate, thanks to the prediction by Chung et al. [27], the expected spectral radius of the normalized Laplacian of a random graph with prescribed degree distribution as

$$
\max _{i>1}\left|1-\nu_{i}\right| \simeq \frac{2}{\sqrt{2\langle k\rangle}} .
$$

Equation (4) generally provides very good estimates for the spectral radius of $\mathcal{L}$ for random networks with Poissonian and power-law degree distributions. By comparing $\nu^{*}$ to the right-hand side of Eq. (4), we finally find that the two transition points are

$$
p_{c}^{+,-} \simeq \frac{1}{2} \pm \frac{1}{\sqrt{2\langle k\rangle}}
$$

In Eq. (5), the solution with the minus sign corresponds to a critical point of the transition between the $\mathcal{B}$ and $\mathcal{I}$ phases, while the solution with the plus sign corresponds to the threshold between the $\mathcal{I}$ and $\mathcal{D}$ phases. As expected, such predictions are in very good agreement with the results of numerical experiments (see Fig. 1).

Next, we relax the former assumption about perfectly aligned degree sequences, and we let $k_{\text {out }}$ correspond to a slightly modified permutation of the sequence $k_{\text {in }}$. Unfortunately, our theory works only for Poisson degree distributions, but the results we obtain also allow us to understand the qualitative behavior of networks with power-law degree distributions. If degrees are random variates extracted from a Poisson distribution, we are able to numerically show that the $i$ th component of the secondsmallest (only in the $\mathcal{D}$ phase) or the largest eigenvector (only in the $\mathcal{B}$ phase) $\mathbf{w}^{*}$ obeys the relation

$$
w_{i}^{*} \simeq c_{i} \frac{p k_{i}^{\text {in }}-(1-p) k_{i}^{\text {out }}}{\sqrt{p k_{i}^{\text {in }}+(1-p) k_{i}^{\text {out }}}},
$$

where $\quad c_{i}=c$ for $i=1, \ldots, N, \quad c_{i}=-c \quad$ for $i=N+1, \ldots, 2 N$, and $c$ is a proper normalization constant. Eq. (6) essentially means that the $i$ th component of the eigenvector $\mathbf{w}^{*}$ is proportional to the difference between the internal and external strengths divided by the square root of the total strength of node $i$ (see Supplemental Material Fig. S1 [25]). The validity of Eq. (6) is testified by the very high linear correlation coefficients that one can measure between the components of the eigenvectors (numerically computed) and the righthand side of Eq. (6). Using Eq. (6) as an ansatz for the solution of the eigenvalue problem, we are able to determine that the eigenvalue of $\mathcal{L}$ associated with the eigenvector $\mathbf{w}^{*}$ of Eq. (6) is given by 

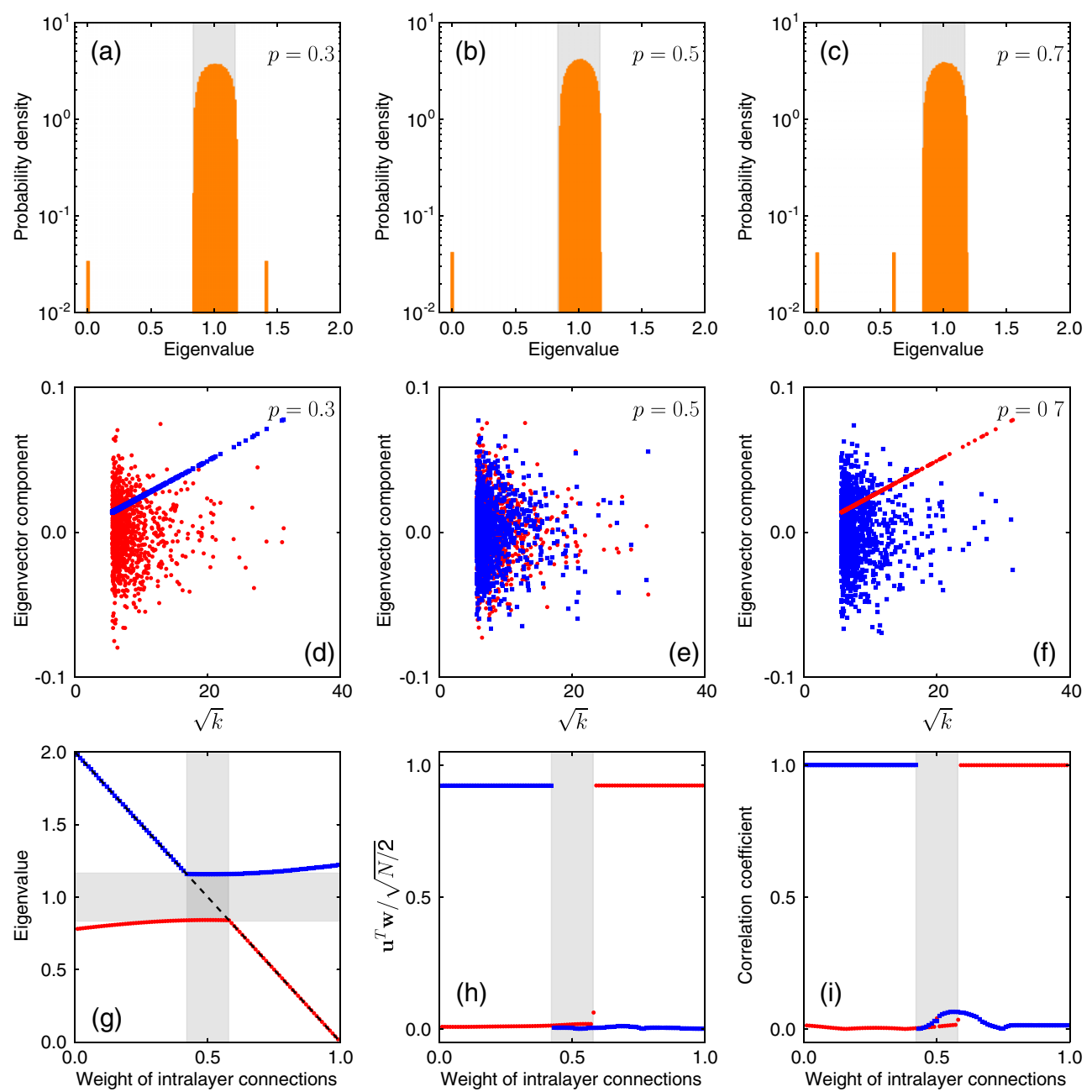

FIG. 1. Abrupt transition in interconnected networks. Spectral analysis of the normalized Laplacian $\mathcal{L}$ of an interconnected network composed of two network layers of size $N=1024$. The degree sequence is composed of integers extracted from a powerlaw probability distribution with exponent $\gamma=2.5$ defined on the support $[32, N]$. Intra- and interlayer degree sequences are perfectly aligned so that the correlation term reads $\xi=\sum_{k^{\text {in }}, k^{\text {out }}} k^{\text {in }} k^{\text {out }} P\left(k^{\text {in }}, k^{\text {out }}\right)=\left\langle k^{2}\right\rangle$. As the weight of the intralayer connections $p$ varies, the system explores different regimes that are clearly visible from the spectrum of $\mathcal{L}$. (a) For $p=0.3$, only the largest eigenvalue $\nu_{2 N}$ is well separated from the rest of the spectrum. The system is said to be in the "bipartite" regime $(\mathcal{B}$ phase). (b) For $p=0.5$, there are no detached eigenvalues. The system is in the "indistinguishable" regime ( $\mathcal{I}$ phase). (c) For $p=0.7$, the secondsmallest eigenvalue $\nu_{2}$ is well separated for the rest of the spectrum. The system is in the "decoupled" regime (D phase). (d) In the $\mathcal{B}$ phase, the components of the largest eigenvector $\mathbf{w}_{2 N}$ corresponding to a single network layer (blue squares) are linearly correlated with the square root of the respective node degrees $\sqrt{k}$, while those of the second-smallest eigenvector $\mathbf{w}_{2}$ (red circles) are not. (e) In the $\mathcal{I}$ phase, there is no correlation between the components of $\mathbf{w}_{2 N}\left(\mathbf{w}_{2}\right)$ and $\sqrt{k}$. (f) In the $\mathcal{D}$ phase, the components of the second-smallest eigenvector $\mathbf{w}_{2}$ are linearly correlated with $\sqrt{k}$, while the components of $\mathbf{w}_{2 N}$ are not. (g) The transition points $p_{c}^{-} \simeq 0.42$ and $p_{c}^{+} \simeq 0.58$ (delimiting the vertical gray band) between the different regimes correspond to the points in which our prediction [Eq. (5), dashed black line] enters the continuous band of the spectrum [Eq. (4), horizontal gray band]. Red circles refer to $\nu_{2}$, while blue squares refer to $\nu_{2 N}$. (h) The sums of the components of $\mathbf{w}_{2}$ (red circles) and $\mathbf{w}_{2 N}$ (blue squares) corresponding to nodes of a single network layer can be used as an order parameter to monitor the transition between the different regimes. ( $\mathbf{u}$ is the vector whose components are all equal to 1.) (i) As noted above, in the $\mathcal{D}$ phase ( $\mathcal{B}$ phase), the components of $\mathbf{w}_{2}$ $\left(\mathbf{w}_{2 N}\right)$ are perfectly correlated with the square root of the node degrees.

$$
\nu^{*} \simeq 1-\frac{\left(2 p^{2}-2 p+1\right)\left\langle k^{2}\right\rangle-2 p(1-p) \xi}{(2 p-1)\left\langle k^{2}\right\rangle}
$$

and thus by the ratio between a quadratic and a linear function of $p$. The function appearing at the denominator depends only on the second moment $\left\langle k^{2}\right\rangle$ of the degree distribution. The function appearing at the numerator, instead, depends on the second moment of the degree distribution and the correlation between intra- and interlayer degrees 

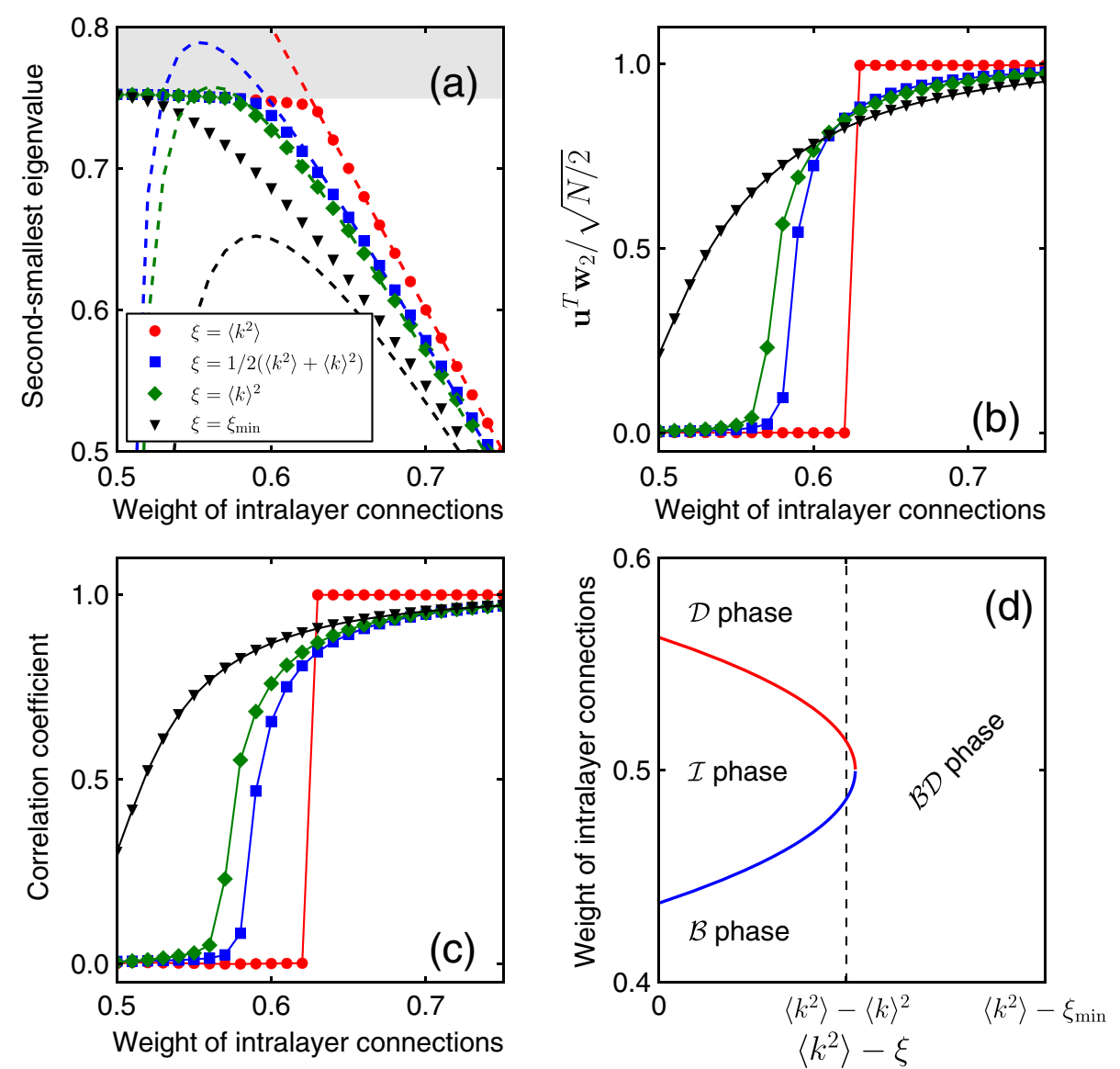

FIG. 2. Phase diagram. We consider interconnected networks composed of two layers of size $N=8192$ with intra- and interlayer degrees obeying a Poisson distribution with average $\langle k\rangle=32$. (a) Second-smallest eigenvalue $\nu_{2}$ as a function of the weight of the intralayer connections $p$. For values of the correlation term $\xi$ in the range $\left[\langle k\rangle^{2},\left\langle k^{2}\right\rangle\right]$, the results of numerical computations (symbols) are in very good agreement with our analytic predictions (dashed lines). The critical point $p_{c}^{+}$is determined as the point for which the dashed lines enter the spectral band (gray area). For $\xi=\xi_{\min }$, our predictions fail to correctly describe how $\nu_{2}$ changes as a function of $p$. (b) As in Fig. 1, we monitor the transition between the $\mathcal{D}$ and the $\mathcal{I}$ phases, by looking at the order parameter $\mathbf{u}^{T} \mathbf{w}_{2} / \sqrt{N / 2}$. As $\xi$ decreases, the change of the order parameter becomes smoother, and the value of the critical point gets closer to $p=0.5$. For $\xi \in\left[\langle k\rangle^{2},\left\langle k^{2}\right\rangle\right]$, the order parameter goes to 0 if $p<p_{c}^{+}$. For $\xi=\xi_{\min }$, instead, the order parameter is even larger than 0 for $p=0.5$. (c) The same behavior described for the order parameter is visible if we monitor the transition through the linear correlation coefficient between the eigenvector components and the right-hand side of Eq. (6). (d) Phase diagram representing the expected behavior of the system in the thermodynamic limit. The red line stands for the critical value $p_{c}^{+}$, while the blue line represents $p_{c}^{-}$[Eq. (9)]. These critical values are plotted as functions of the quantity $\left\langle k^{2}\right\rangle-\xi$, ranging between 0 and $\left\langle k^{2}\right\rangle-\xi_{\min }$. As a reference, the dashed line represents the point at which $\xi=\left\langle k^{2}\right\rangle$. Although the curves corresponding to $p_{c}^{+}$and $p_{c}^{-}$do not intersect, for values of the correlation term $\xi$ that are sufficiently low, their analytic continuations suggest the disappearance of the $\mathcal{I}$ phase and the appearance of a supercritical regime where both the bipartite and the decoupled phases are present (i.e., the $\mathcal{B D}$ phase).

$$
\xi=\sum_{k^{\text {in }}, k^{\text {out }}} k^{\text {in }} k^{\text {out }} P\left(k^{\text {in }}, k^{\text {out }}\right) .
$$

By comparing Eq. (7) with the expected spectral radius of Eq. (4), we finally arrive at the prediction of the critical points

$$
p_{c}^{+,-} \simeq \frac{1}{2}\left[1 \pm \frac{\frac{2}{\sqrt{2\langle k\rangle}}\left\langle k^{2}\right\rangle+\sqrt{\xi^{2}+\left\langle k^{2}\right\rangle^{2}\left(\frac{2}{\langle k\rangle}-1\right)}}{2\left(\left\langle k^{2}\right\rangle+\xi\right)}\right]
$$

When the term inside the square root of Eq. (9) is larger than 0 , we have an intersection between $\nu^{*}$ and the rest of the spectrum of $\mathcal{L}$. This intersection indicates the presence of an abrupt transition between the corresponding phases. Strictly speaking, these eigenvalues do not intersect in finite-size random networks, as predicted by the Wignervon Neumann noncrossing rule [28,29]; however, the abrupt nature of the transition already appears clear for networks of moderately large size. When the term in the square root of Eq. (9) is equal to 0, the nontrivial eigenvalue $\nu^{*}$ touches the rest of the spectrum of $\mathcal{L}$ 

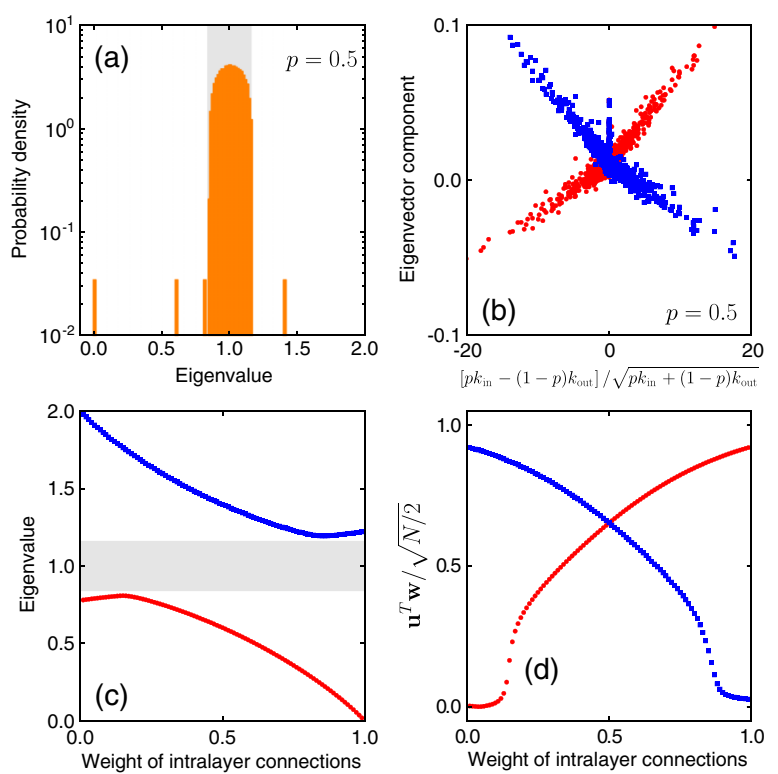

FIG. 3. Supercritical regime. Spectral analysis of the normalized Laplacian $\mathcal{L}$ for an interconnected network with a degree sequence identical to the one analyzed in Fig. 1. In this case, however, intra- and interlayer degree sequences are aligned in such a way that the correlation term is $\xi \simeq 1 / 2\left(\left\langle k^{2}\right\rangle+\langle k\rangle^{2}\right)$. (a) For $p=0.5$, the system is in the $\mathcal{B D}$ phase, where both the eigenvalues $\nu_{2}$ and $\nu_{2 N}$ are well separated from the other eigenvalues of $\mathcal{L}$. (b) The components of both eigenvectors $\mathbf{w}_{2}$ and $\mathbf{w}_{2 N}$ are correlated with the right-hand side of Eq. (6). (c) The eigenvalues $\nu_{2}$ (red circles) and $\nu_{2 N}$ (blue squares) touch the continuous band tangentially. (d) The order parameter clearly shows the presence of the supercritical regime, where both the $\mathcal{B}$ and $\mathcal{D}$ phases are simultaneously present, for a wide range of values of $p$. Only for very low (large) values of $p$, the system is in the pure $\mathcal{B}$ phase $(\mathcal{D}$ phase).

tangentially at $p_{c}$. In this case, the corresponding transition becomes continuous. The nonexistence of a real solution in Eq. (9) reveals instead the absence of a crossing between $\nu^{*}$ and the rest of the spectrum even in infinite-size networks and thus is the sign of a dramatic change in the behavior of the system.

Equations (7) and (9) show that the entire scenario is characterized by two main factors. On one end, the degree distribution, with its first two moments, plays a fundamental role for the determination of the various phases. On the other hand, the degree distribution alone is not able to explain the behavior of the system. At parity of moments, in fact, the features of the system can still be drastically changed by the correlation term defined in Eq. (8). To better understand how the various possibilities are regulated by the interplay between the correlation term $\xi$ and the moments of the degree distribution, we vary the correlation term by regulating the alignment between the intra- and interlayer degree sequences $k_{\text {in }}$ and $k_{\text {out }}$. If the order of the sequences $k_{\text {in }}$ and $k_{\text {out }}$ is identical, $\xi=\left\langle k^{2}\right\rangle$, and Eqs. (7) and (9) correctly reduce to Eqs. (3) and (5), respectively. If, from this initial alignment, we randomly shuffle a selected portion of pairs of entries in the interlayer degree sequence $k_{\text {out }}$, we decrease the value of $\xi$. In particular, if we randomly mix all entries, then $k_{\text {out }}$ corresponds to a random permutation of $k_{\text {in }}$ and the correlation term reads $\xi=\langle k\rangle^{2}$. Finally, if the entries of $k_{\text {in }}$ are ordered in ascending (descending) order, while those of $k_{\text {out }}$ are sorted in descending (ascending) order, the correlation term reaches the minimum value $\xi_{\min }$ [30]. When $\xi$ is in the range $\left[\langle k\rangle^{2},\left\langle k^{2}\right\rangle\right]$, the term in the square root of Eq. (9) is strictly larger than 0: All regimes exist, and the corresponding phase transitions are discontinuous (see Fig. 2). At the same time, the phase diagram suggests that a further decrement of $\xi$ leads to a drastic mutation in the features of the system. For lower values of $\xi$, the analytic continuation of our predictions tells us that (i) phase transitions change their nature and become continuous and (ii) the $\mathcal{I}$ phase is no longer present and leaves space to a hybrid $\mathcal{B D}$ phase where the system is simultaneously in the bipartite and decoupled regimes. The presence of this hybrid phase appears evident even by looking at the spectral properties of the normalized Laplacian of finite-size systems: For $\xi=\xi_{\min }$ and $p=0.5$, the order parameters associated with the $\mathcal{B}$ and $\mathcal{D}$ phases are, in fact, simultaneously larger than 0 (see Fig. 2 and Supplemental Material Fig. S2 [25]).

The previous effect is amplified when we consider networks whose degrees obey a power-law degree distribution $P(k) \sim k^{-\gamma}$, with $\gamma<3$. Differently from the case of Poissonian networks, in fact, the correlation term $\xi$ has a much larger range of variability. Although the ansatz of Eq. (6) breaks down for scale-free networks, and thus the entire analytic approach is not working, Eq. (9) still tells us what we should expect to see: Unless the correlation term $\xi$ is very close to its maximal value $\left\langle k^{2}\right\rangle$, the term in the square root is negative; this fact means that the $\mathcal{B D}$ phase is reached already for little variations from the perfect alignment between the intra- and interlayer sequences $k_{\text {in }}$ and $k_{\text {out }}$. Our qualitative predictions are indeed supported by numerical experiments, as shown in Fig. 3 and Supplemental Material Fig. S3 [25]. For $p=0.5$, the presence of the hybrid $\mathcal{B D}$ phase is testified by the fact that the eigenvalues $\nu_{2}$ and $\nu_{2 N}$ are simultaneously well separated from the other eigenvalues of $\mathcal{L}$, and the associated eigenvectors $\mathbf{w}_{2}$ and $\mathbf{w}_{2 N}$ have components that are very well correlated with the right-hand side of Eq. (6). The mixing between the two degree sequences causes a mismatch between intra- and interlayer hubs that do not correspond to the same nodes as in the case of perfectly aligned degree sequences. From the structural point of view, the total disappearance of the so-called indistinguishable phase can be interpreted as the absence of a buffer of structural safety. Scale-free networks seem, therefore, to be constantly at risk of being potentially catastrophic failures. There are, however, also dramatic dynamical consequences. While for high values of $\xi$ there is a neat 

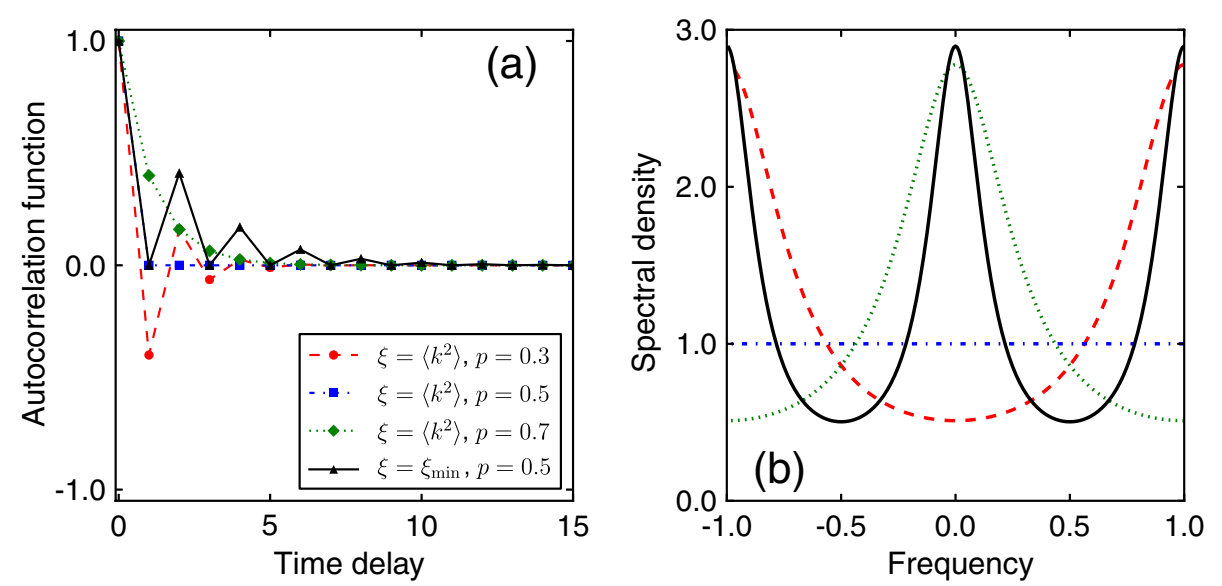

FIG. 4. Random-walk dynamics. We consider two interconnected networks composed of $N=8192$ nodes. Degrees are extracted from a power-law distribution with exponent $\gamma=2.5$ defined on the support $[32, N]$. We monitor the trajectory of a random walker moving on these systems for different values of the correlation term $\xi$ and different values of the weight of the intralayer connections $p$. (a) Autocorrelation function $R(\tau)=\langle x(t) x(t+\tau)\rangle$ as a function of the time delay $\tau$. In the definition of $R(\tau), x(t)=+1$ if the random walker does not change network layer at time $t$, and $x(t)=-1$ otherwise. (b) Spectral density $S(\omega)$ of the autocorrelation functions of (a). Intralayer movements are characterized by the presence of a peak of $S(\omega)$ at $\omega=0$, while interlayer jumps are emphasized by the presence of peaks at $\omega= \pm 1$.

distinction between the $\mathcal{B}$ and $\mathcal{D}$ phases and a random walker moves in the network performing either intra- or interlayer diffusion, in the $\mathcal{B D}$ phase, instead, both motions happen simultaneously (Fig. 4). Such an ability makes the walker faster in the exploration of the entire system (See Supplemental Material Fig. S4 [25]).

To summarize, the spectral properties of the normalized Laplacian of random interconnected networks show that these systems face a scenario that is much richer than the one that is valid for isolated networks. Depending on the combination of basic structural properties-degree distribution, degree correlation, and strength difference between intra- and interlayer connections-topological and dynamical phase transitions associated with the spectrum of the normalized Laplacian can be either discontinuous or continuous, and different regimes can disappear or even coexist. Our predictions allow us, somehow, to divide the space of structural parameters into regions where the "classical" theory of isolated network holds and those in which interdependent networks behave in an anomalous manner. As a final remark, we would like to stress an appealing analogy between our findings and the typical thermodynamic behavior of any substance, e.g., water, carbon dioxide, and methane. In normal conditions, a substance changes its phase from liquid to gas in a discontinuous manner. However, above its critical temperature and pressure, the substance ceases to exhibit a distinct liquid- or gaslike state, and it becomes a so-called supercritical fluid [31]. Since their properties can be fine-tuned, supercritical fluids have many industrial and scientific applications. We believe that the possibility to drive interconnected networks to supercritical regimes could also be used in a fruitful way to better design and control real-world networked systems.

The author thanks A. Arenas, C. Castellano, and A. Flammini for discussions and comments on the subject of this article.

[1] S. V. Buldyrev, R. Parshani, G. Paul, H. E. Stanley, and S. Havlin, Catastrophic Cascade of Failures in Interdependent Networks, Nature (London) 464, 1025 (2010).

[2] M. Szell, R. Lambiotte, and S. Thurner, Multirelational Organization of Large-Scale Social Networks in an Online World, Proc. Natl. Acad. Sci. U.S.A. 107, 13636 (2010).

[3] M. Barthélemy, Spatial Networks, Phys. Rep. 499, 1 (2011).

[4] F. Radicchi and A. Arenas, Abrupt Transition in the Structural Formation of Interconnected Networks, Nat. Phys. 9, 717 (2013).

[5] S. N. Dorogovtsev, A. V. Goltsev, and J. F. F. Mendes, Critical Phenomena in Complex Networks, Rev. Mod. Phys. 80, 1275 (2008).

[6] R. Parshani, S. V. Buldyrev, and S. Havlin, Interdependent Networks: Reducing the Coupling Strength Leads to a Change from a First to Second Order Percolation Transition, Phys. Rev. Lett. 105, 048701 (2010).

[7] J. Gao, S. V. Buldyrev, S. Havlin, and H. E. Stanley, Robustness of a Network of Networks, Phys. Rev. Lett. 107, 195701 (2011).

[8] J. Gao, S. V. Buldyrev, H. E. Stanley, and S. Havlin, Networks Formed from Interdependent Networks, Nat. Phys. 8, 40 (2012).

[9] S.-W. Son, G. Bizhani, C. Christensen, P. Grassberger, and M. Paczuski, Percolation Theory on Interdependent 
Networks Based on Epidemic Spreading, Europhys. Lett. 97, 16006 (2012).

[10] F. Chung, Spectral Graph Theory (American Mathematical Society, 1996).

[11] S. Jianbo and M. Jitendra, Normalized Cuts and Image Segmentation, IEEE Trans. Pattern Anal. Mach. Intell. 22, 888 (1997).

[12] M. Rosvall and C. T. Bergstrom, Maps of Random Walks on Complex Networks Reveal Community Structure, Proc. Natl. Acad. Sci. U.S.A. 105, 1118 (2008).

[13] M. Cavers, S. Fallat, and S. Kirkland, On the Normalized Laplacian Energy and General Randic Index $R_{-1}$ of Graphs, Linear Algebra Appl. 433, 172 (2010).

[14] P. G. Doyle and L. Snell, Random Walks and Electric Networks (Mathematical Association of America, Washington, D.C., 1984).

[15] D. J. Klein and M. Randić, Resistance Distance, J. Math. Chem. 12, 81 (1993).

[16] H. C. Berg, Random Walks in Biology (Princeton University Press, Princeton, NJ, 1993).

[17] S. Brin and L. Page, The Anatomy of a Large-Scale Hypertextual Web Search Engine, Comput. Netw. ISDN Syst. 30, 107 (1998).

[18] E. A. Codling, M. J. Plank, and S. Benhamou, Random Walk Models in Biology, J. R. Soc. Interface 5, 813 (2008).

[19] B. G. Malkiel, A Random Walk Down Wall Street (Norton, New York, 1973).

[20] G. H. Weiss, Aspects and Applications of the Random Walk (Random Materials and Processes) (North-Holland, Amsterdam, 1994).
[21] M. Faccin, T. Johnson, J. Biamonte, S. Kais, and P. Migdał, Degree Distribution in Quantum Walks on Complex Networks, Phys. Rev. X 3, 041007 (2013).

[22] L. Lovász, in Combinatorics, Paul Erdős is Eighty, edited by D. Miklós, V. T. Sós, and T. Szőnyi (János Bolyai Mathematical Society, Budapest, Hungary, 1996), Vol. 2, p. 1-46.

[23] I. B. Schwartz and L. Billings, Fluctuations Induced Almost Invariant Sets, Naval Research Laboratory Technical Report No. NRL-MR-6790-06-9012, 2006.

[24] S. Fortunato, Community Detection in Graphs, Phys. Rep. 486, 75 (2010).

[25] See Supplemental Material at http://link.aps.org/ supplemental/10.1103/PhysRevX.4.021014 for explicit calculations and additional figures.

[26] S. Blundell and K. M. Blundell, Concepts in Thermal Physics (Oxford University Press, New York, 2006).

[27] F. Chung, L. Lu, and V. Vu, Spectra of Random Graphs with Given Expected Degrees, Proc. Natl. Acad. Sci. U.S.A. 100, 6313 (2003).

[28] J. von Neumann and E. Wigner, No Crossing Rule, Z. Phys. 30, 467 (1929).

[29] P. D. Lax, Linear Algebra and Its Applications (Wiley-Interscience, New York, 2007), 2nd ed.

[30] Z. Darćzy, Rearrangement Inequalities, Publ. Math. Debrecen 20, 273 (1973).

[31] M. McHugh and V. Krukonis, Supercritical Fluid Extraction: Principles and Practice (Butterworth-Heinemann, London, 1986). 